$\Rightarrow$ LIPID BILAYERS

\section{Atomistic complexity}

at physio-
logically
relevant
temperatures
we can identify
detailed
molecular
interactions
that people
speculate
underlie
some of the
complexity
of biological
membranes

The cell membrane is a complex, heterogeneous system in which different phase domains coexist. This complexity poses several challenges for both experimental and theoretical studies of lipid bilayer organization. Ruo-Xu Gu, Svetlana Baoukina and Peter Tieleman from the University of Calgary have now used a newly developed atomistic approach to gain detailed information on phase separation in the cell membrane.

The domains in the cell membrane are thought to regulate the function of membrane proteins. Their formation arises as a result of the different lipids having different gel-liquid transition temperatures. But studying them is challenging as a result of their small size $(10-200 \mathrm{~nm})$ and rapid dynamics. Experimental and computational studies are usually performed on model membranes that include only two or three components, for example, a low-melting lipid such as 1,2-dioleoylsn-glycero-3-phosphocholine (DOPC), a high-melting one such as 1,2-dipalmitoyl-sn-glycero-3phosphocholine (DPPC) and cholesterol.

"Experimental studies using fluorescence microscopy and NMR can inform us on the phase diagram of model membranes, as well as the

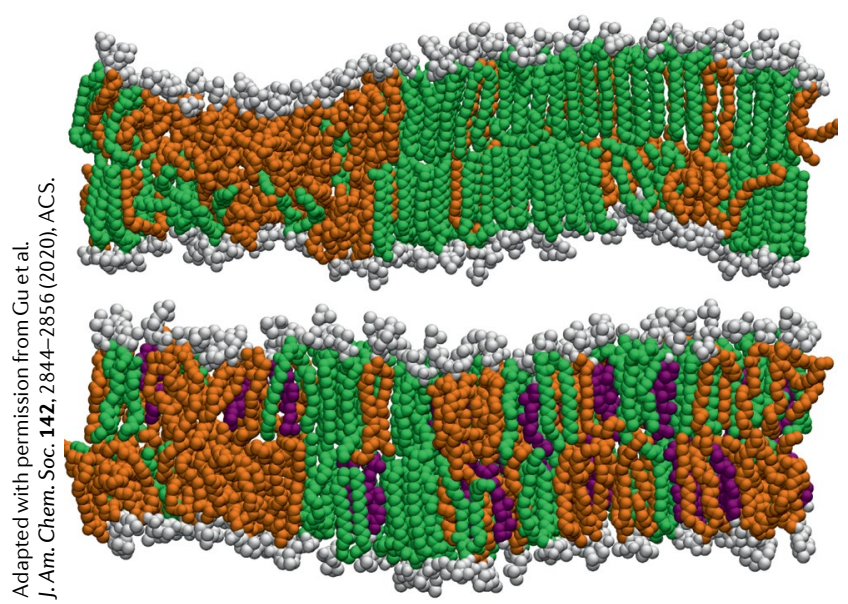

properties of the domains. But they provide limited information on the domain coexistence and interactions at the interface between different domains at the molecular level. Furthermore, experimental studies at room temperature reveal the formation of micron-sized domains, but these are much larger than those anticipated to be found at physiological temperatures," says Gu. The possible nanometric size of these domains at physiological temperatures challenges their experimental study even more, making the aid of computer simulation essential.

Coarse-grained molecular dynamics is the go-to approach for the simulation of large complex systems. By defining small molecular fragments as 'grains' in the model it is possible to simulate microsecondlong trajectories of reasonably large (nanometre-sized) complex systems at reasonable computational costs.

Coarse-grained models, of course, risk the loss of important atomistic detail. A coarse-grained simulation might underestimate orientational ordering of lipid tails, for example, and thus the entropic contribution to phase transition. The enthalpic contribution would then need to compensate to explain the behaviour, leading ultimately to incorrect conclusions about the driving force of phase separation. In this work, $\mathrm{Gu}$ and co-workers have used an atomistic force field, which uses fewer approximations and results in a more realistic physical model.

Phase transitions from disorder to gel in lipid membranes were simulated for binary mixtures (DPPC:DOPC) and ternary mixtures (DPPC:DOPC:cholesterol) in the temperature range $310-270 \mathrm{~K}$ over a $10 \mu$ s time frame. The binary mixture evolves from a homogeneous, disordered liquid phase at $310 \mathrm{~K}$ to coexisting liquid and gel phases at $290-280 \mathrm{~K}$ and coexisting liquid and rippled phases below $270 \mathrm{~K}$. "This is a very surprising result. According to the phase diagram of one-component membranes, a ripple phase is an intermediate state between the liquid and the gel phase. The origin of the different phase diagram for binary mixtures is still not fully clear. This result highlights our limited knowledge of the ordered domains in binary mixture, which may be worth further investigation," explains $\mathrm{Gu}$.

For the ternary mixture, the homogenous liquid phase at $310 \mathrm{~K}$ changes to the coexistence of disordered and ordered liquid phases, to the gel-liquid coexistence with the lowering of the temperature. The calculated phase diagram is in agreement with the experimental observations. However, these simulations provide molecular understanding on the role of small cholesterol clusters, which increase order and decrease mobility in the disordered phase and have the opposite effect in the more ordered phases.

"Our simulations are currently among the most extensive to provide an atomistic picture of the behaviour of a complex lipid mixture, while trying to link as closely as possible to known experimental data. It is unlikely (I'd say impossible) that experimental techniques will ever come close to the amount of structure and dynamic detail on bilayers of this complexity," remarks Tieleman. "We find more complexity in the cases that we looked at than the literature suggests. We also show that at physiologically relevant temperatures we can identify detailed molecular interactions that people speculate underlie some of the complexity of biological membranes. I would continue in this area with more targeted hypotheses on the role of specific lipids that are common in biological membranes and hope these simulations inspire both new experiments and further exploration and development of computational models," he concludes.

Gabriella Graziano

ORIGINAL ARTICLE Gu, R.-X., Baoukina, S. \& Tieleman, D. P. Phase separation in atomistic simulations of model membranes. J. Am. Chem. Soc. 142, 2844-2856(2020) 